\title{
Application of Potential Theory to Steady Flow Past Two Cylinders in Tandem Arrangement
}

\author{
Yangyang Gao, ${ }^{1,2}$ Danielle S. Tan, ${ }^{3}$ Zhiyong Hao, ${ }^{4}$ Xikun Wang, ${ }^{5}$ and Soon Keat Tan ${ }^{6}$ \\ ${ }^{1}$ Ocean College, Zhejiang University, Hangzhou 310058, China \\ ${ }^{2}$ The State Key Laboratory of Fluid Power Transmission and Control, Zhejiang University, Hangzhou 310058, China \\ ${ }^{3}$ Cornell University, Ithaca, NY 14853, USA \\ ${ }^{4}$ College of Logistics Engineering, Shanghai Maritime University, Shanghai 201306, China \\ ${ }^{5}$ Maritime Research Centre and School of Civil and Environmental Engineering, College of Engineering, \\ Nanyang Technological University, Singapore, Singapore 639798 \\ ${ }^{6}$ Nanyang Environment and Water Research Institute and School of Civil and Environmental Engineering, \\ Nanyang Technological University, Singapore, Singapore 639798
}

Correspondence should be addressed to Yangyang Gao; yygao@zju.edu.cn

Received 25 August 2013; Revised 31 December 2013; Accepted 14 January 2014; Published 5 March 2014

Academic Editor: Shihua Li

Copyright (C) 2014 Yangyang Gao et al. This is an open access article distributed under the Creative Commons Attribution License, which permits unrestricted use, distribution, and reproduction in any medium, provided the original work is properly cited.

\begin{abstract}
The wake flow patterns associated with flow past a cylinder and a cylinder-pair in tandem configuration are revisited, compared, and evaluated with respect to the streamline patterns generated based on potential flow theory and superposition of various potential flow elements. The wakes, which are vortex shedding in the lee of the cylinder(s), are reproduced by placing pairs of equal but opposite circulation elements in the potential flow field. The strength of the circulation elements determines the size of the vortices produced. The streamline patterns of flow past a pair of unequal cylinders in tandem configuration provide an indirect means to establish the threshold condition for the wake transition from that of a single bluff body to alternating reattachment behavior. This threshold condition is found to be a function of the diameter ratio, $d / D$ (diameters $d$ and $D, d \leq D$ ), spacing ratio, $L / D$ (centre-tocentre distance, $L$, to cylinder diameter, $D)$, and equivalent incident flow speed, $U$. A unique functional relationship $f(L / D, d / D$, $U$ ) of this threshold condition is established.
\end{abstract}

\section{Introduction}

Flow past a cylinder and two circular cylinders in tandem configuration are common occurrences in many engineering applications, such as mooring lines, riser, and bundled cylinders at offshore installations, bridge piers, and tube bundles in heat exchangers. The wake flow patterns in the lee of a single cylinder are well understood, while there is still much unknown in the case of a cylinder-pair in tandem configuration. A cylinder-pair may be of different diameters, with equal diameters being the special case. Many attempts have been made to categorize the flow behavior behind a pair of tandem cylinders with equal diameter (downstream of the first cylinder and in the lee of the cylinder-pair) (Igarashi [1, 2]; Ljungkrona and Sundén [3]; Sumner et al. [4]; Lin et al. [5]; Xu and Zhou [6]; Zhou and Yiu [7];
Carmo et al. [8, 9]; Sumner [10]). Zdravkovich [11] identified three basic types of interference based on the range of flow Reynolds number (Re): (i) single bluff body (composite) behavior at small centre-to-centre spacing (type 1); (ii) shear layer reattachment at intermediate spacing (type 2); and (iii) Karman vortex shedding from each cylinder independently at larger spacing (type 3). There is but a handful of studies on unequal cylinder-pairs (Igarashi [12]; Mahbub Alam and Zhou [13]; Zhao et al. [14, 15]; Gao et al. [16, 17]). Under certain cross-flow speed ( $U$ or Re) and depending on the spacing ratio $L / D$ and diameter ratio $d / D$, these three types of flow interference can also be observed, as illustrated in Figure 1. Here, for a fixed incident flow speed $U(=1)$ and $d / D=2 / 3$, the reattachment and alternating vortex shedding patterns in the wake of the upstream cylinder begin to appear at $L / D=2.4$, with the cylinder-pair behaving like a single 

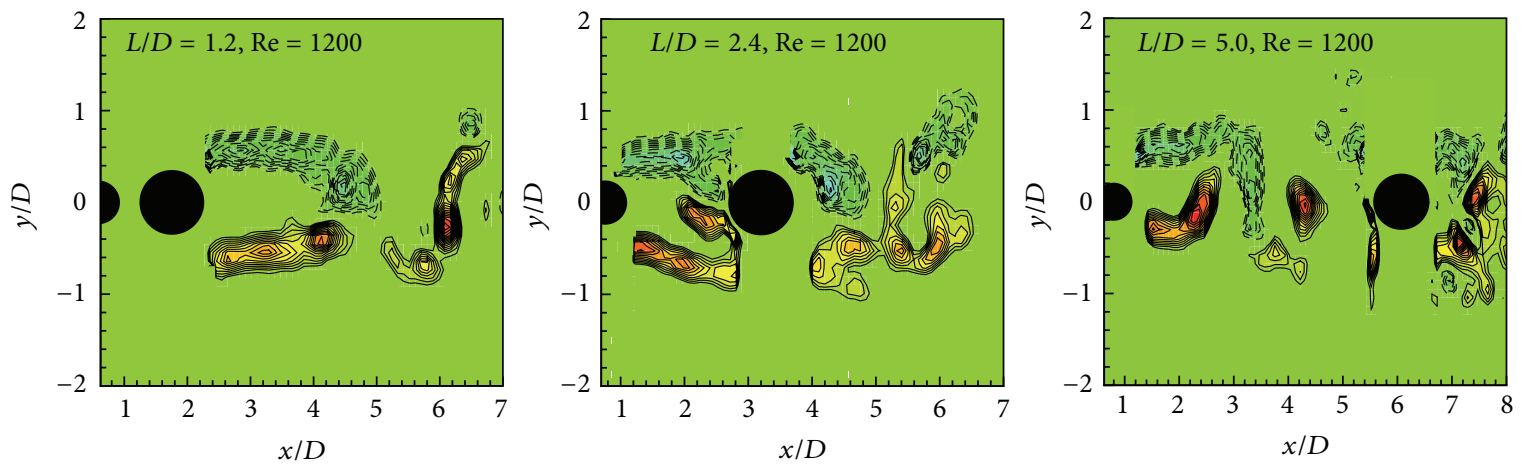

(a)

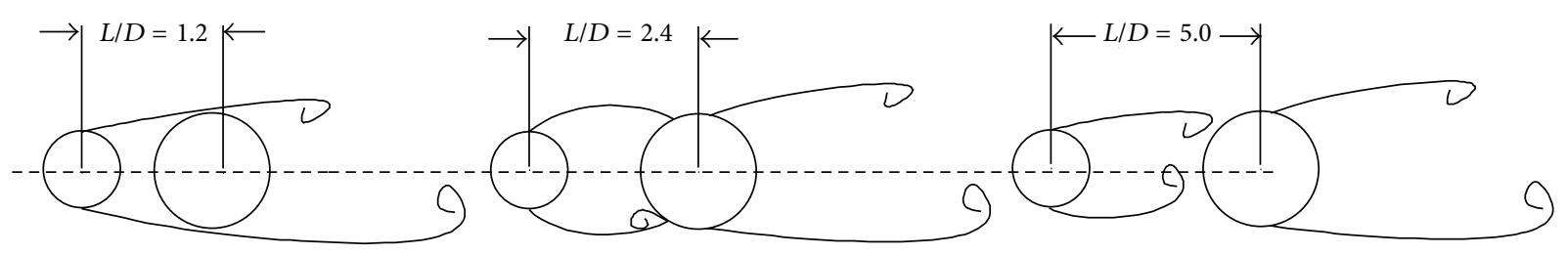

(b)

Figure 1: Instantaneous flow patterns around two tandem cylinders with $d / D=2 / 3$ at different $L / D$, for a fixed incident flow speed (Re).

(a) Laboratory observation based on particle image velocimetry (PIV) and (b) schematic flow structure.

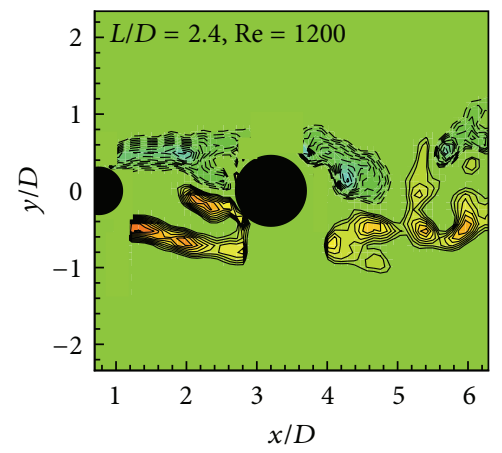

(a)

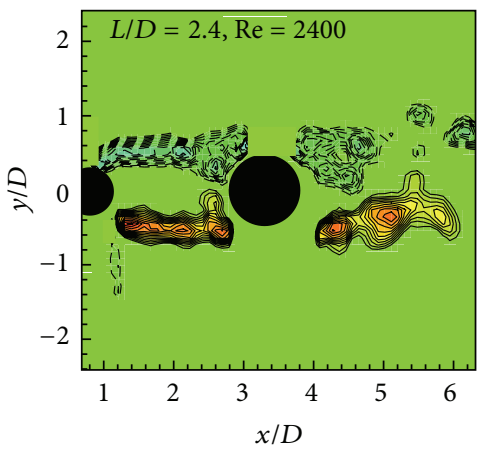

(b)

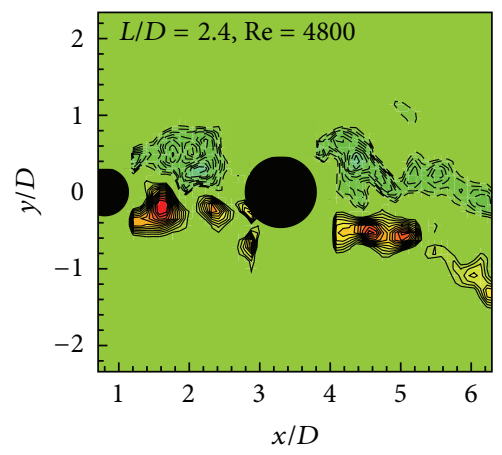

(c)

FIgURE 2: Instantaneous flow patterns around two tandem cylinders at $L / D=2.4$ for a fixed $d / D$ but different Re.

bluff body for $L / D<2.4$. This observation indicates that the value $L / D=2.4$ corresponds to a threshold condition that demarcates the end of single bluff body behavior and the beginning of alternating vortices in the region between the two cylinders. For $L / D=2.4$ and $d / D=2 / 3$, all three types of interference can be observed consecutively with increasing incident flow speed (Reynolds number), as shown in Figure 2, which indicates that the Reynolds number has significant effect on determination of the threshold condition.

Of the three types of interference, most engineering applications would prefer the bundled cylinders to behave as a single bluff body. Therefore, it is desirable to establish the threshold condition below which the single bluff body behavior is observed. The authors are not aware of any analytical expression for this threshold value of $L / D$, or said expression, as a function of $d / D$ and speed of the cross-flow $U$, or Re. Reported spacing ratios $L / D$ at which type 1 and type 2 interference take place are listed in Table 1 . It can be seen from this table that the upper limit of the spacing ratio for type 1 interference is sensitive to the Reynolds number and is consistent with Igarashi's [1] suggestion.

The authors noticed that there are uncanny similarities between the streamline patterns generated using potential theory for a pair of doublets placed at different centre-tocentre spacing ratios and the flow patterns shown in Figures 1 and 2. It appears that one could draw an analogy between potential flow about a pair of doublet and flow past a cylinderpair in tandem arrangement. Using potential flow theory as a tool to aid in the derivation of analytical/numerical solutions for high Reynolds number flows is not new. This technique has been applied in many studies on surface waves, the wake around a ship hull, and ship-to-ship interactions, where the fluid is assumed to be steady, incompressible, inviscid, and irrotational in the far field (Millward et al. [18]; Shahjada Tarafder and Suzuki [19]; Ahmed and Guedes Soares [20]). Potential flow around multiple cylinders has also 
TABLE 1: Summary of spacing ratio ranges for single bluff body and reattachment flow patterns behind two tandem circular cylinders.

\begin{tabular}{|c|c|c|c|c|}
\hline Author & Cylinder diameter: $d, D$ & $\operatorname{Re}$ & $(L / D)_{\mathrm{I}}$ & $(L / D)_{\mathrm{II}}$ \\
\hline Igarashi [1] & $d=D$ & $8700-5.2 \times 10^{4}$ & $1.03-1.91$ & $2.09-3.53$ \\
\hline Zdravkovich [11] & $d=D$ & Not given & $1-1.2 \sim 1.8$ & $1.2 \sim 1.8-3.4 \sim 3.8$ \\
\hline Ljungkrona and Sundén [3] & $d=D$ & $(1.0-1.2) \times 10^{4}$ & 1.25 & $2.0,4.0$ \\
\hline $\mathrm{Xu}$ and Zhou [6] & $d=D$ & $800-4.2 \times 10^{4}$ & $1.0-2.0$ & $2.0-3.0$ \\
\hline Zhou and Yiu [7] & $d=D$ & 7000 & 1.3 & $2.5,4.0$ \\
\hline Gao et al. [16] & $d<D$ & $1200-4800$ & 1.2 & 2.4 \\
\hline
\end{tabular}

$(L / D)_{\mathrm{I}}$ : centre-to-centre spacing ratio range for single bluff body pattern.

$(L / D)_{\text {II }}$ : centre-to-centre spacing ratio range for reattachment flow pattern.

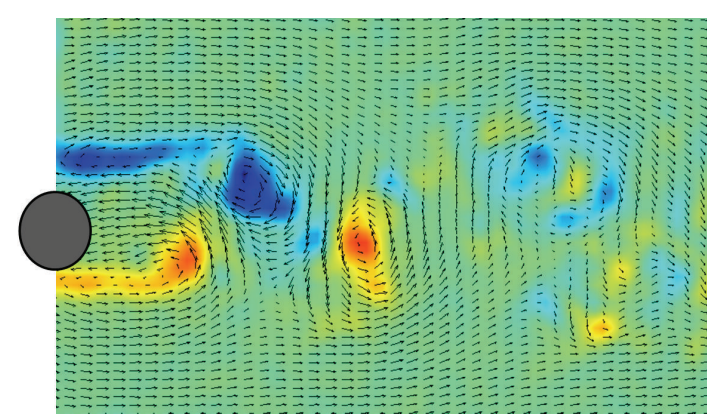

(a) $\operatorname{Re}=1200, U=0.1 \mathrm{~m} / \mathrm{s}$

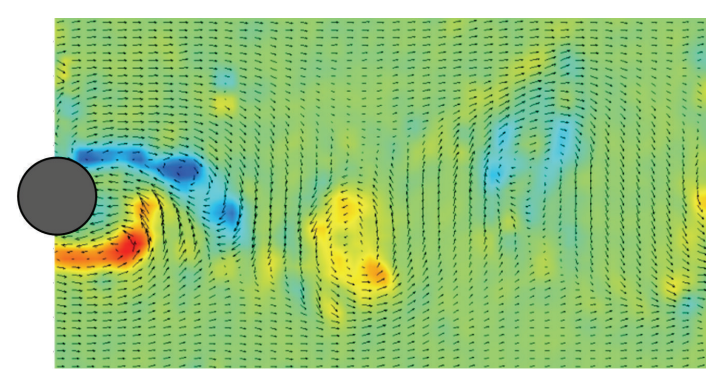

(b) $\operatorname{Re}=4800, U=0.2 \mathrm{~m} / \mathrm{s}$

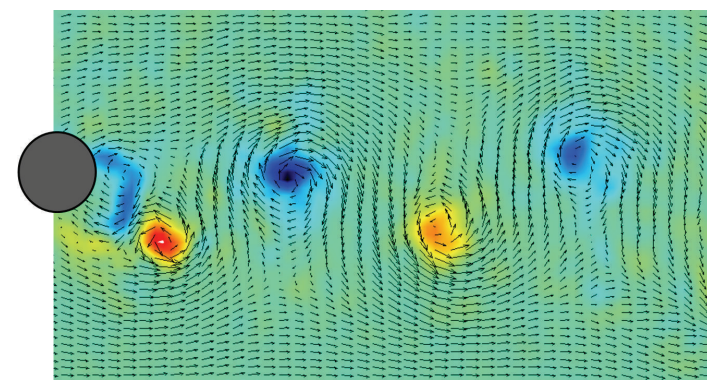

(c) $\operatorname{Re}=7200, U=0.4 \mathrm{~m} / \mathrm{s}$

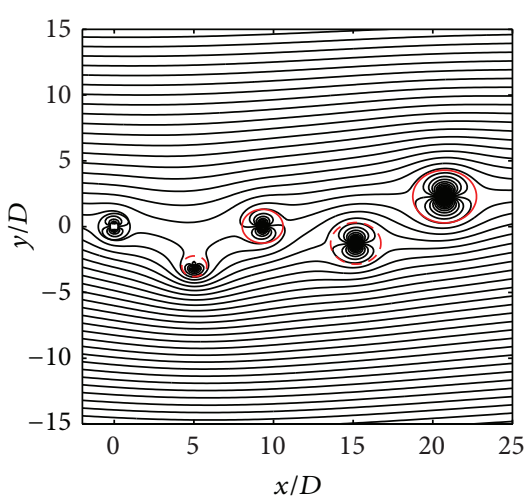

(d)

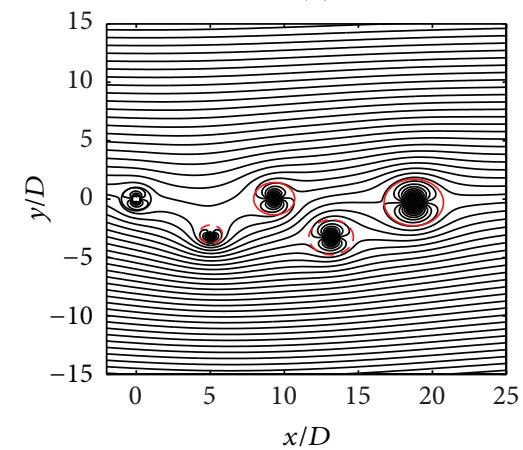

(e)

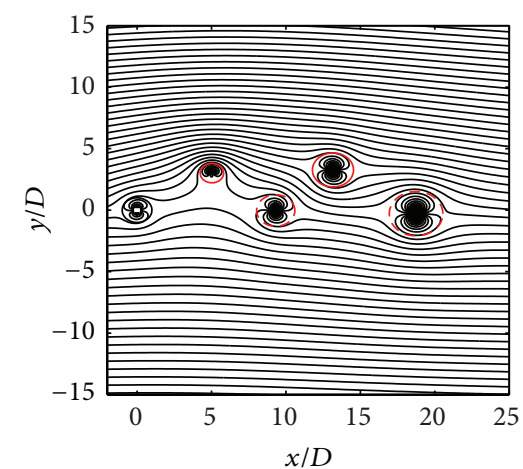

(f)

FIGURE 3: Comparison of instantaneous flow patterns around a single cylinder at different Re obtained using PIV (a)-(c), and streamline flow patterns based on potential flow (d)-(f). 
been investigated by many researchers to derive analytical solutions for potential flow past multiple cylinders, as in Johnson and McDonald [21], Burton et al. [22], and Crowdy [23]. Considering the analogous patterns of potential flow streamlines and the flow patterns behind cylinder-pair in tandems, the obvious question arises as to whether one could deduce from these patterns a precise threshold condition analytically and account for the onset of type 2 and type 3 interferences.

For this purpose, the authors drew on the potential flow theory approach to generate streamline patterns for the following cases:

(1) wake flow in the lee of a single cylinder;

(2) flow patterns in the lee of a cylinder-pair, as a function of spacing ratio and diameter ratio, with the aim of determining the threshold spacing ratio for the onset of streamline patterns similar to that of type 2 and type 3 interference.

\section{Derivation of Streamline Patterns Based on Potential Flow Theory}

2.1. Flow Past a Single Cylinder. When an ideal fluid flows past a single circular cylinder, the streamlines and velocity potentials form the same pattern as that of a doublet placed in a constant uniform flow as suggested by Acheson [24]. Thus, the complex potential function for a uniform flow past a single cylinder may be expressed as

$$
W(z)=V\left(z+\frac{1}{z}\right)
$$

where $V$ is the flow velocity parallel to the $x$-axis and $z$ is the complex variable. For flow past a cylinder, the uniform flow field is represented by the flow velocity $V$. The streamline patterns obtained from the potential theory based on $1 / V$ are found to be appropriate. Hence, the uniform flow field is represented by $U=1 / V$ in this study, and the potential function is expressed as $\psi(U)=\psi(1 / V)$.

The complex flow past a circular cylinder over the $z$ plane can be constructed by superposition of potential flow elements. In this case, the authors use a uniform flow and vortex element with certain circulation strength. Here, the vortex element in the potential flow is represented by a rotating body of a certain diameter $D$ and circulation strength $\Gamma$. The circulation is either clockwise or counterclockwise depending on the sign assigned to $\Gamma$. Consequently, the complex potentials for flow past a single circular cylinder can be visualized as being the resultant of a uniform flow and a rotating vortex element and can be written as

$$
W(z)=U z+\frac{U a^{2}}{z}+\frac{i \Gamma}{2 \pi} \ln z .
$$

Consider the wake flow patterns captured using the particle image velocimetry (PIV) technique and shown in Figures 3(a)-3(c); the gyros in the flow are vortices of certain strength in the flow field. These gyros could be represented

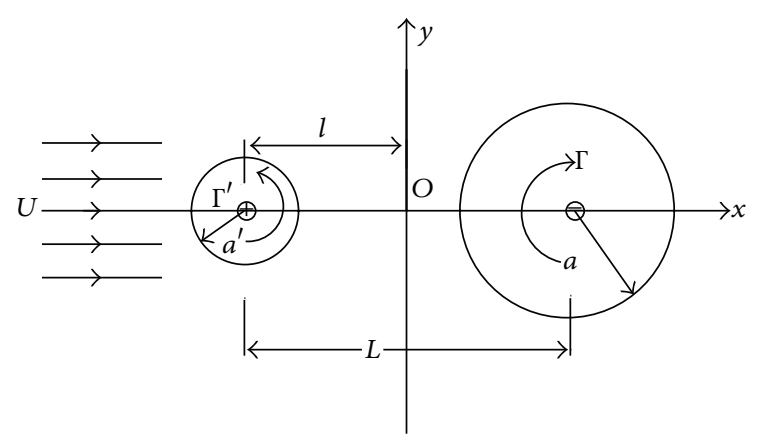

Figure 4: A schematic diagram of two cylinders with unequal diameters in tandem arrangement. " $O$ " is the origin. The size of the cylinder is reflected by the strength of circulation. Here, $D=2 a$ and $d=2 a^{\prime}$, for $a>a^{\prime}$.

by placing a circulation element of appropriate strength in the flow, with the corresponding streamlines presented in Figures $3(\mathrm{~d})-3(\mathrm{f})$. The potential equation corresponding to these streamlines is as follows:

$$
\begin{gathered}
W(z)=F_{1}(z)+F_{2}(z)+F_{3}(z)+F_{4}(z)+F_{5}(z) \\
=U z+U a^{2}\left[\frac{\beta_{1}}{z-L_{1}}+\frac{\beta_{2}}{z-L_{2}}+\frac{\beta_{3}}{z-L_{3}}\right. \\
\left.\quad+\frac{\beta_{4}}{z-L_{4}}+\frac{\beta_{5}}{z-L_{5}}\right] \\
+\frac{i \Gamma}{2 \pi}\left[\ln \left(z-L_{1}\right)^{\alpha_{1}}+\ln \left(z-L_{2}\right)^{\alpha_{2}}+\ln \left(z-L_{3}\right)^{\alpha_{3}}\right. \\
\left.+\ln \left(z-L_{4}\right)^{\alpha_{4}}+\ln \left(z-L_{5}\right)^{\alpha_{5}}\right],
\end{gathered}
$$

where $F_{j}(z)$ represents the complex potential for each circulation element $j(j=1,2,3,4,5) . \beta_{j}$ and $\alpha_{j}$ represent the diameter ratio and circulation strength ratio, respectively, for each element. It can be seen from Figure 3 that the principal features of the wake flow patterns could be reproduced by using circulation elements to represent the gyros.

While the patterns generated reflect physically meaningful flow phenomenon, it is recognized that the above approach requires prior knowledge of the location and strength of the circulations.

2.2. Flow Past a Cylinder-Pair in Tandem Configuration. For flow past two tandem cylinders as shown in Figure 4, the complex flow can be constructed by superposition of a uniform flow, two vortex elements, and two pertinent circulation strengths with opposite signs. Consider the diameter ratio of the upstream to downstream cylinders is $\beta=a^{\prime} / a$ ( $a^{\prime}$ and $a$ are the radii of the upstream and downstream cylinders, resp.) and circulation at the upstream cylinder is $\Gamma^{\prime}=\beta \Gamma$ (where $\Gamma$ is the circulation of the downstream cylinder). The complex potentials for the upstream and downstream 


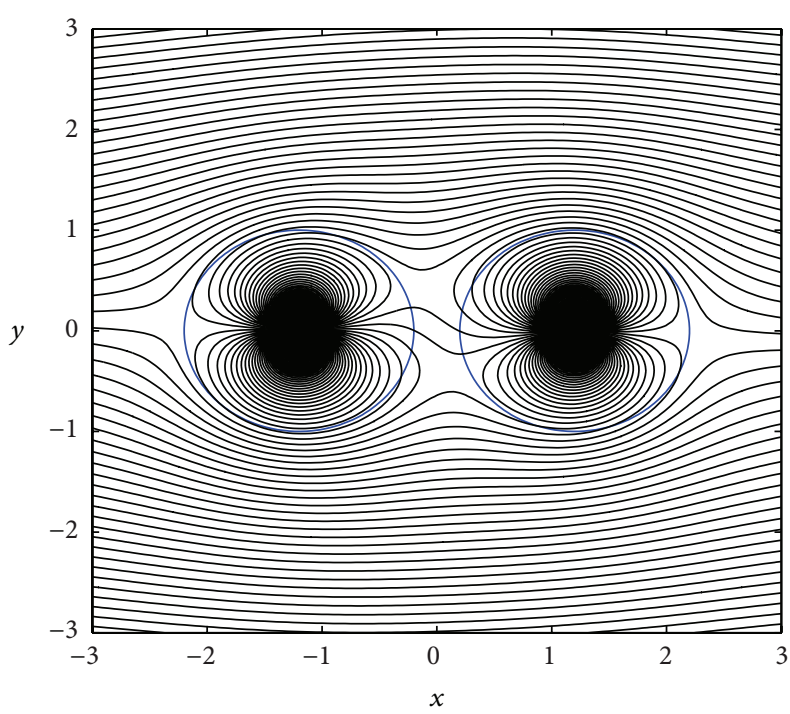

(a)

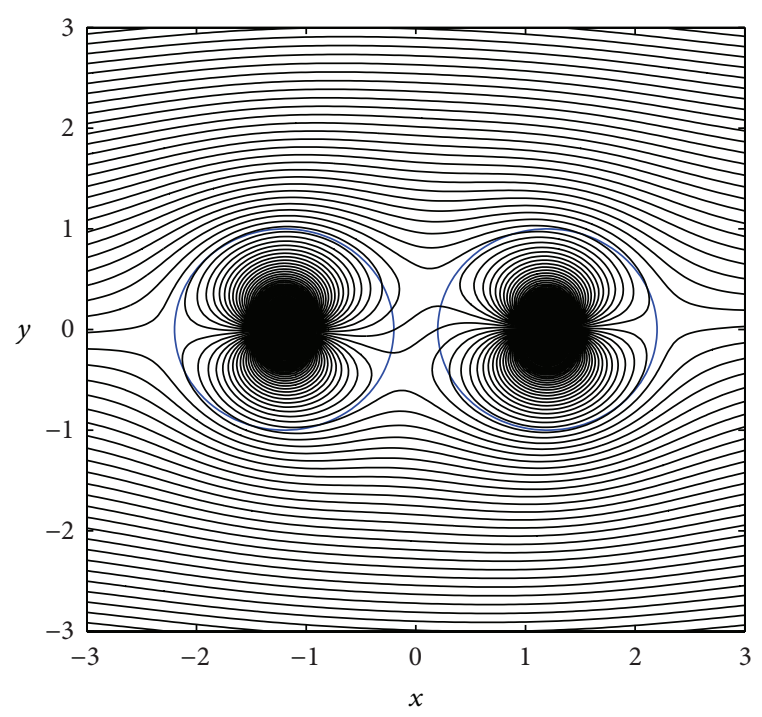

(b)

FIGURE 5: Streamlines around two cylinders with equal diameter in tandem at $L / D=1.2$. (a) Upstream cylinder with positive vortex; (b) upstream cylinder with negative vortex.

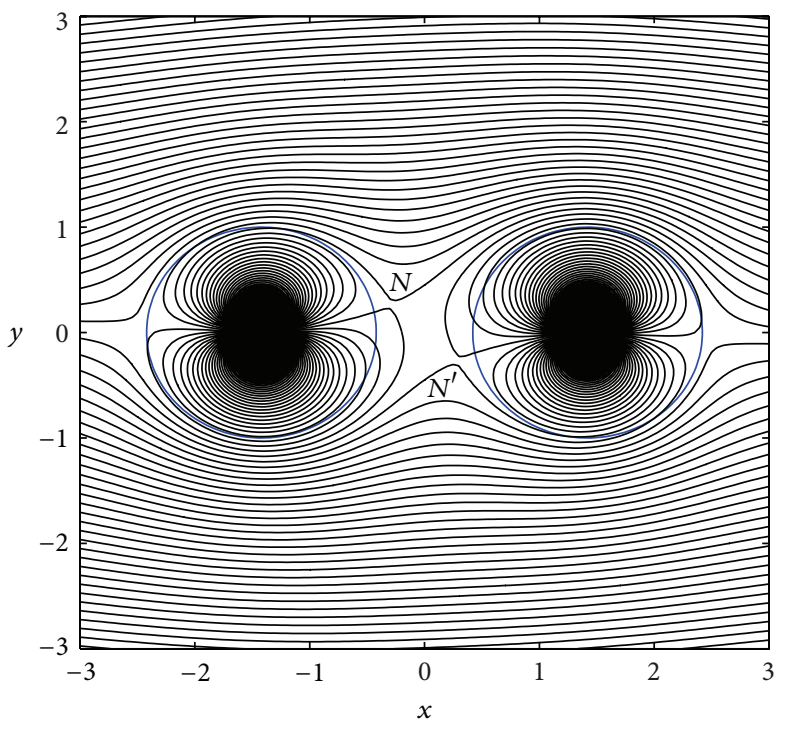

(a)

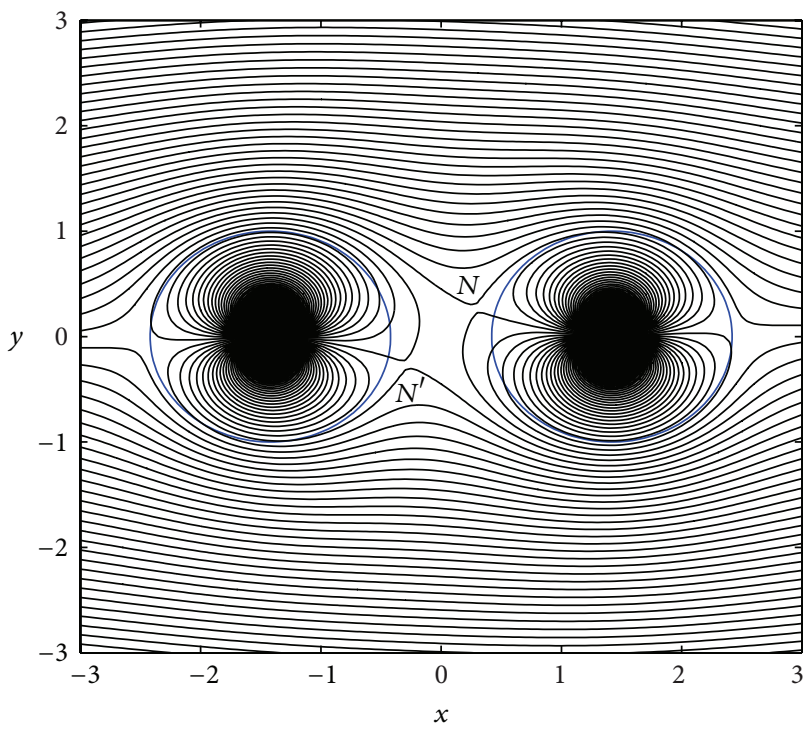

(b)

FIGURE 6: Streamlines around two tandem cylinders at $L / D=1.42$. (a) Upstream cylinder with positive vortex; (b) upstream cylinder with negative vortex.

cylinders located, respectively, at $z=-l$ and $z=l$ may be written as

$$
\begin{aligned}
& F_{1}(z)=U z+\frac{U(\beta a)^{2}}{(z+l)}-\frac{i \beta \Gamma}{2 \pi} \ln (z+l), \\
& F_{2}(z)=U z+\frac{U a^{2}}{(z-l)}+\frac{i \Gamma}{2 \pi} \ln (z-l) .
\end{aligned}
$$

Then, the resultant complex potential $W(z)$ for two tandem cylinders with unequal diameters in a steady flow (see Figure 4) is

$$
\begin{aligned}
W(z)= & F_{1}(z)+F_{2}(z) \\
= & U z+\frac{U a^{2}}{(z-l)}+\frac{U(\beta a)^{2}}{(z+l)}+\frac{i \Gamma}{2 \pi} \ln (z-l) \\
& -\frac{i \beta \Gamma}{2 \pi} \ln (z+l)
\end{aligned}
$$




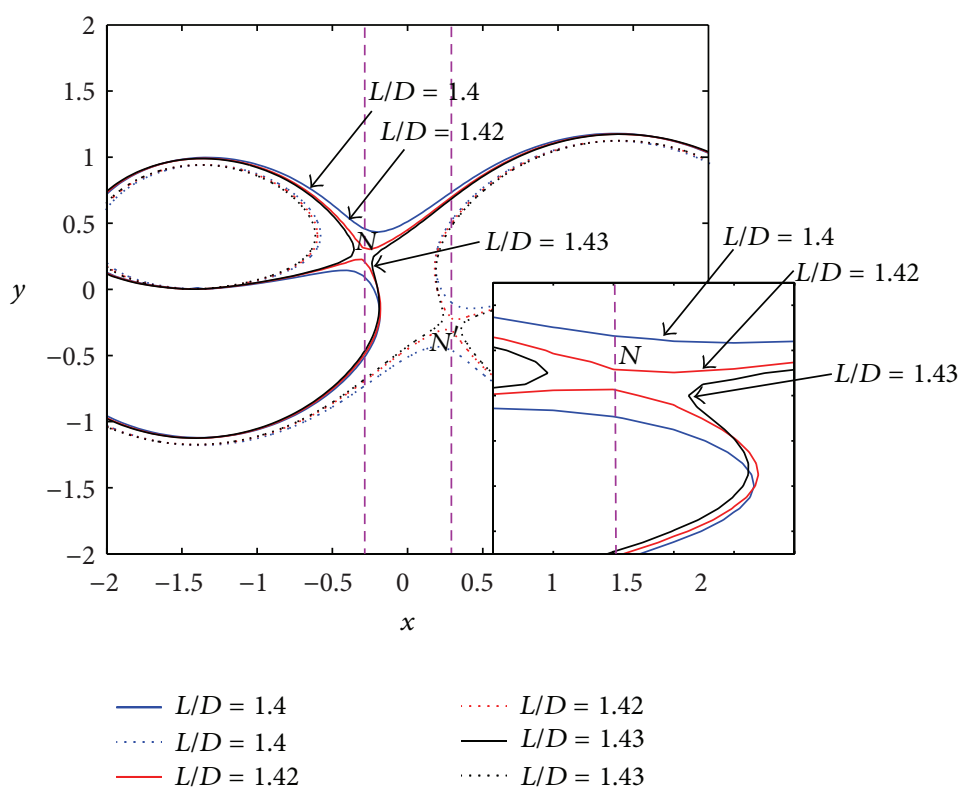

FIgURE 7: The closest streamlines to the surface of two tandem cylinders at different $L / D$; the solid line represents the positive value and dotted line represents the negative value. A magnified view is shown at the lower right corner.

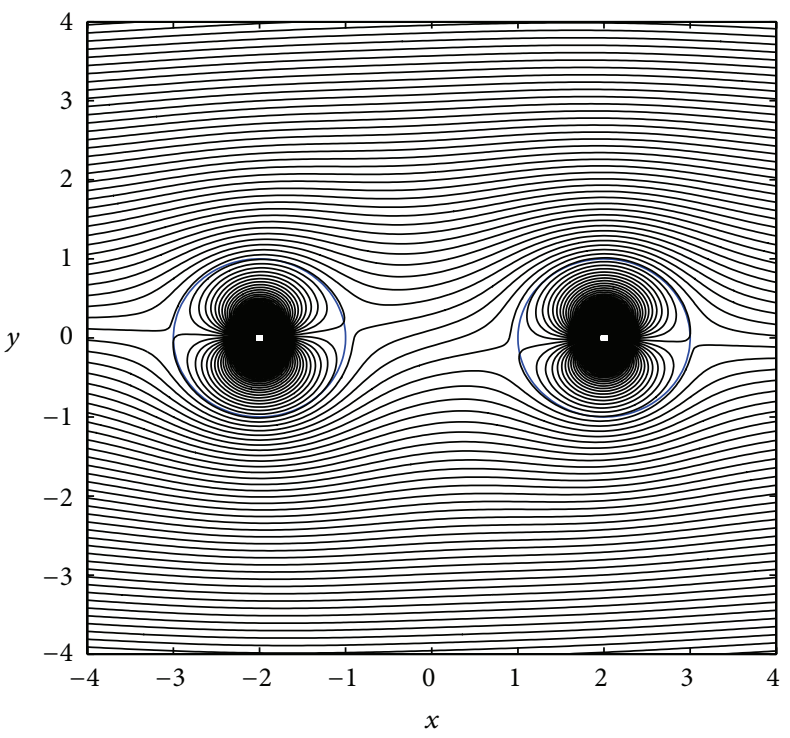

(a)

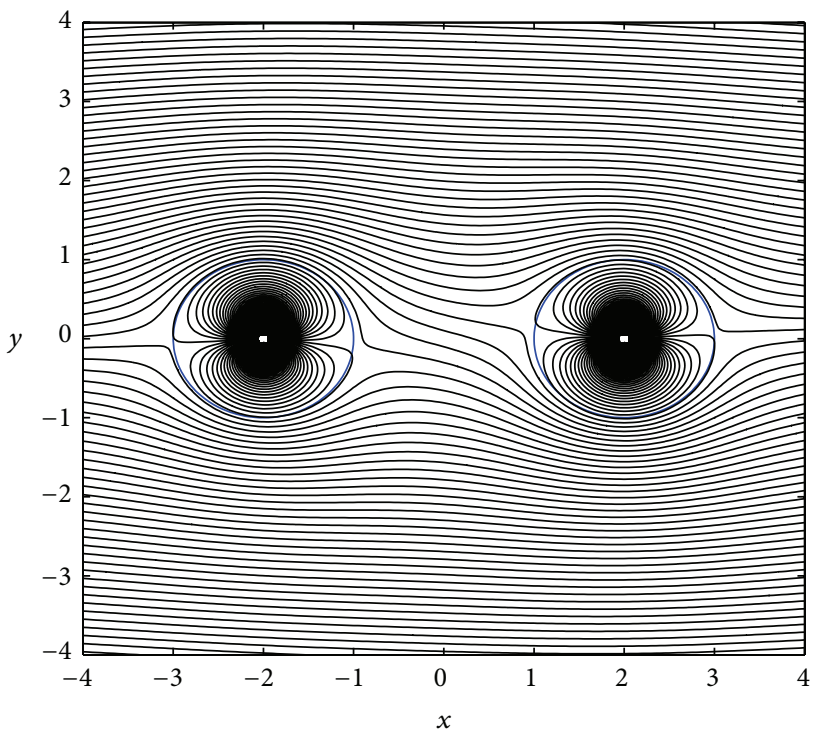

(b)

Figure 8: Streamlines around two tandem cylinders at $L / D=2.0$. (a) Upstream cylinder with positive vortex; (b) upstream cylinder with negative vortex.

$$
\begin{aligned}
= & U\left[z+\frac{a^{2}}{(z-l)}+\frac{(\beta a)^{2}}{(z+l)}\right] \\
& +i \frac{\Gamma}{2 \pi} \ln [(z-l)-\beta \ln (z+l)] .
\end{aligned}
$$

Putting $z=x+i y$ into (5), the complex potential may be expressed as

$$
\begin{aligned}
W(z) & =F_{1}(z)+F_{2}(z) \\
& =U x+U a^{2}\left[\frac{(x-l)}{(x-l)^{2}+y^{2}}+\frac{\beta^{2}(x+l)}{(x+l)^{2}+y^{2}}\right]
\end{aligned}
$$




$$
\begin{gathered}
-\frac{\Gamma}{2 \pi}\left\{\arg [(x-l)+y i]-\arg [(x+l)+y i]^{\beta}\right\} \\
+i\left\{U y-U a^{2}\left[\frac{y}{(x-l)^{2}+y^{2}}+\frac{\beta^{2} y}{(x+l)^{2}+y^{2}}\right]\right. \\
\left.+\frac{\Gamma}{4 \pi} \ln \frac{(x-l)^{2}+y^{2}}{\left[(x-l)^{2}+y^{2}\right]^{\beta}}\right\} .
\end{gathered}
$$

The velocity function $\psi$ and velocity potential $\phi$ for two tandem cylinders are obtained as

$$
\begin{aligned}
\psi= & U y-U a^{2}\left[\frac{y}{(x-l)^{2}+y^{2}}+\frac{\beta^{2} y}{(x+l)^{2}+y^{2}}\right] \\
& +\frac{\Gamma}{4 \pi} \ln \frac{(x-l)^{2}+y^{2}}{\left[(x+l)^{2}+y^{2}\right]^{\beta}}, \\
\phi= & U x+U a^{2}\left[\frac{(x-l)}{(x-l)^{2}+y^{2}}+\frac{\beta^{2}(x+l)}{(x+l)^{2}+y^{2}}\right] \\
& -\frac{\Gamma}{2 \pi}\left\{\arg [(x-l)+y i]-\arg [(x+l)+y i]^{\beta}\right\} .
\end{aligned}
$$

The equations in (7) are the potential and streamline functions that describe the potential and streamlines of a pair of unequal cylinder in tandem configuration, and they are functions of incident flow velocity $U$, spacing ratio $L / D$, and diameter ratio $d / D$.

When $\beta=a^{\prime} / a=1.0$, that is, the two tandem cylinders have equal diameter, the velocity function $\psi_{E}$ and velocity potential $\phi_{E}$ for the two cylinders may be simplified as follows:

$$
\begin{aligned}
\psi_{E}= & U y-U a^{2}\left[\frac{y}{(x-l)^{2}+y^{2}}+\frac{y}{(x+l)^{2}+y^{2}}\right] \\
& +\frac{\Gamma}{4 \pi} \ln \frac{(x-l)^{2}+y^{2}}{(x+l)^{2}+y^{2}}, \\
\phi_{E}= & U x+U a^{2}\left[\frac{(x-l)}{(x-l)^{2}+y^{2}}+\frac{(x+l)}{(x+l)^{2}+y^{2}}\right] \\
& -\frac{\Gamma}{2 \pi}\{\arg [(x-l)+y i]-\arg [(x+l)+y i]\} .
\end{aligned}
$$

\section{Results and Discussions}

3.1. Cylinder-Pair with Equal Diameter (Special Case of $V=$ $1 / U=1.0)$. Based on the potential flow theory, and for $V=1 / U=1$, the authors analyzed the streamlines around two cylinders with equal diameter in tandem at $L / D=1.2$ as

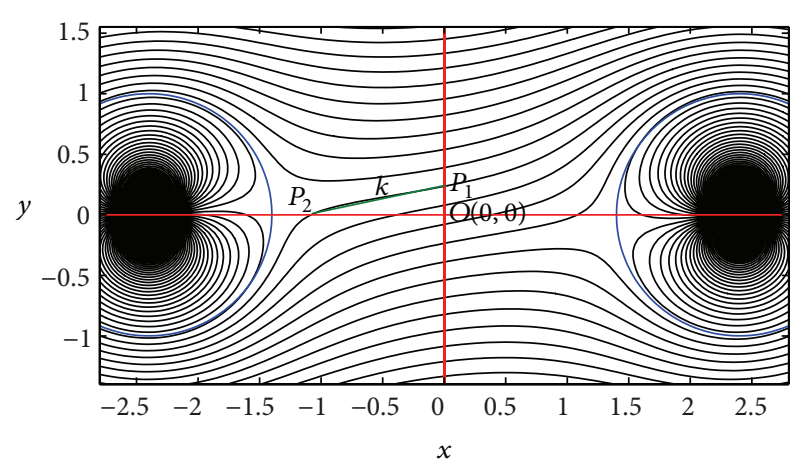

FIGURE 9: Identification of slope of the streamline nearest to the surface of upstream cylinder.

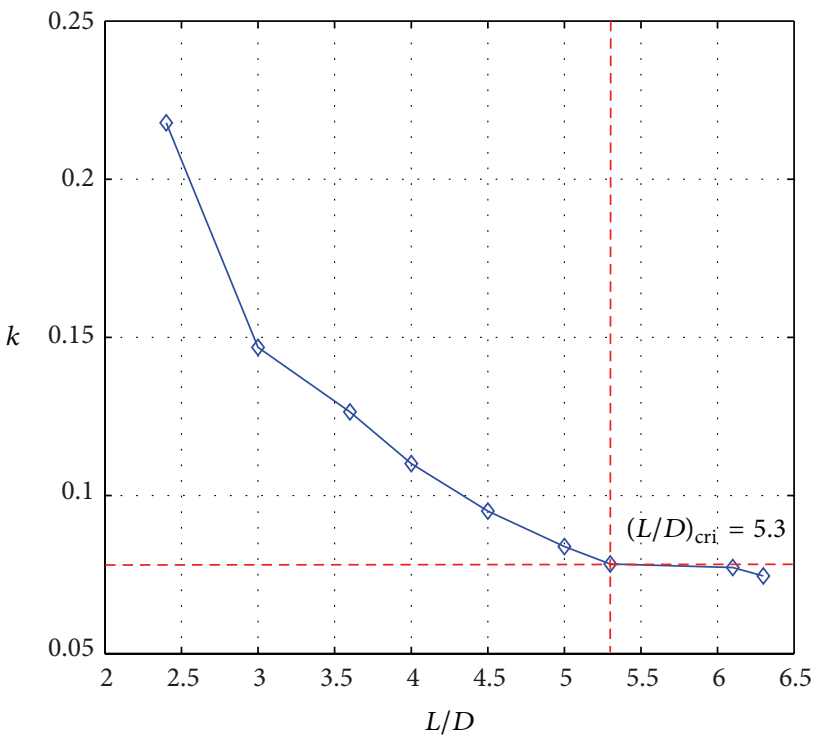

FIGURE 10: The relationship between slope $k$ and $L / D$ at intermediate spacing ratios.

shown in Figure 5. Figure 5(a) shows the results in the case of an upstream cylinder with a positive vortex and downstream cylinder with a negative vortex, while Figure 5(b) shows the opposite circulation arrangement. It can be seen that, for a small spacing ratio, the two cylinders behave as a single bluff body. The streamline patterns suggest that the flow engulfs the two doublets wholly, and the flow patterns are in good agreement with those observed in experiments (Igarashi [2]; Zdravkovich [11]).

As can be seen in Figure 6, when $L / D$ is increased to 1.42, the two cylinders still behave as a single bluff body. In Figure 6, $N$ indicates the innermost point of the upper streamline which is attached to the cylinder with the positive vortex. The counterpart of $N, N^{\prime}$, lies on the streamline attached to the cylinder with the negative vortex. Figure 7 is an enlarged view of the streamlines in the vicinity of $N$ (and $N^{\prime}$ ), showing the nearest streamlines around the surface of two cylinders for different $L / D$ and the pair of tongue-like features near the cylinders. The solid line represents the upper 


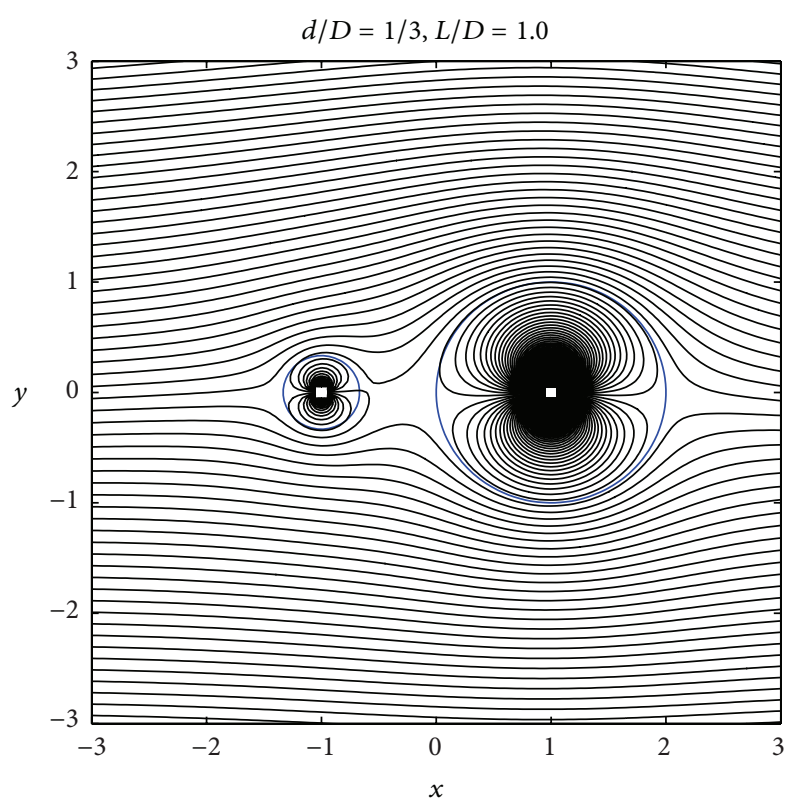

(a)

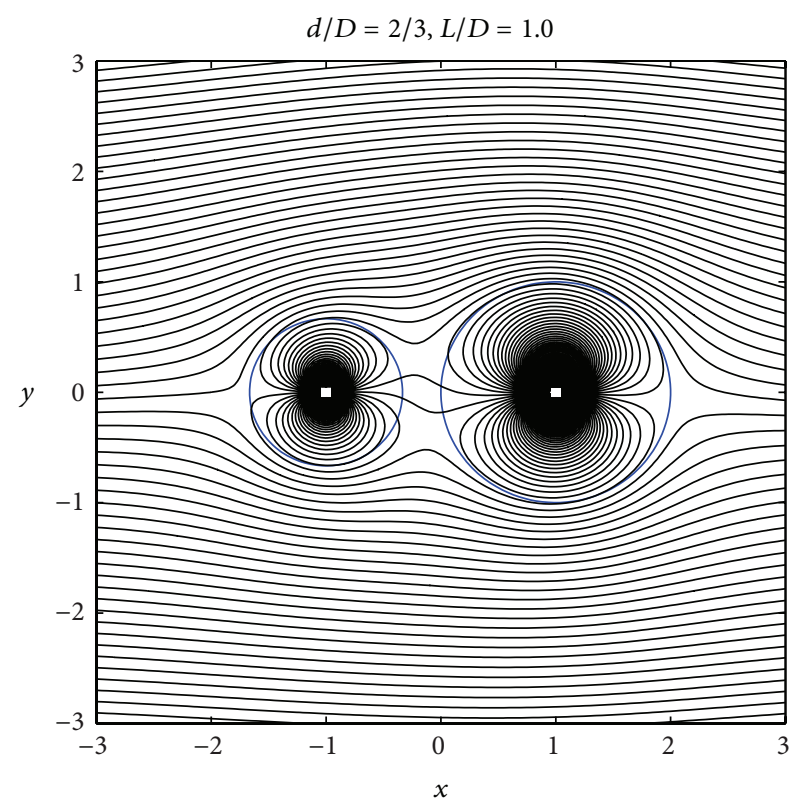

(b)

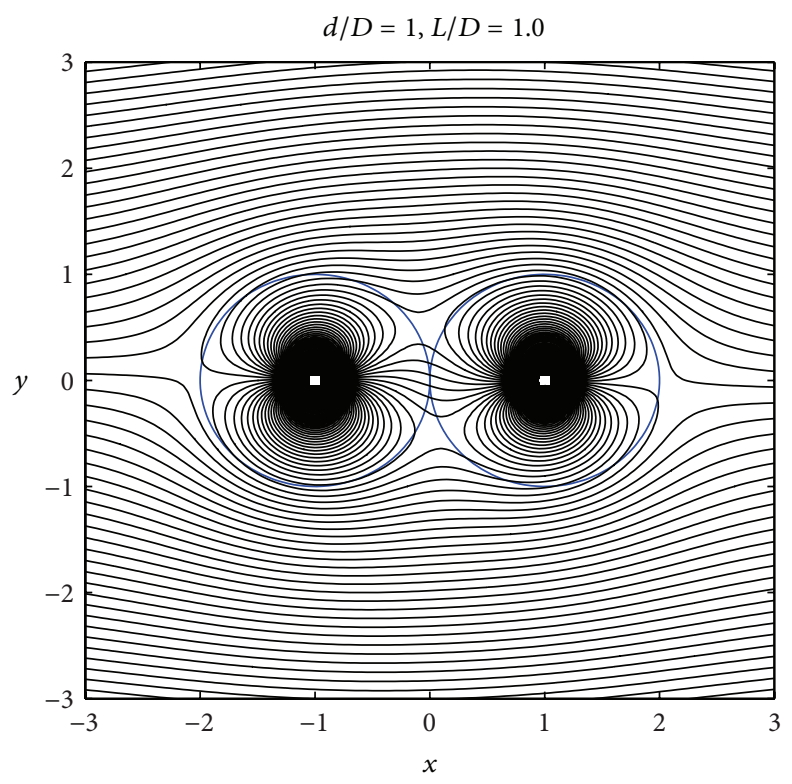

(c)

FIGURE 11: Streamlines around two tandem cylinders with different diameter ratios $d / D$ at $L / D=1.0$.

set of streamlines and the dotted line represents the lower set. A sudden change in the flow patterns occurs when $L / D$ is in the neighborhood of 1.42 . At $L / D=1.43$, the tongue disappears and is replaced by streamlines that cut diagonally across the gap between the two cylinders and cross the axis joining the two cylinder centres, indicating the end of type 1 interference and the onset of type 2 interference.

When $L / D>1.42$, it can be seen from Figure 8 that the streamline patterns in the gap between the two cylinders are distinctly different from those at smaller $L / D$ (see Figures 5 and 6). In Figures 8(a) and 8(b), the streamlines cross diagonally in the region between the two cylinders. This is an interesting observation, suggesting that the alternating reattachment is the result of synchronous alternating circulation about the upstream and downstream cylinders. These intersecting patterns clearly depict the alternating attachment of the shear layers between the cylinders observed at intermediate spacing ratios as reported in the literature (Gao et al. [17]), where the vortices shed from the upstream cylinder reattach on the upper/lower surface of the downstream cylinder in an alternating clockwise and counterclockwise manner. From the potential flow theory formulation, one can see that the phenomenon of alternating reattachment on the downstream cylinder is the 


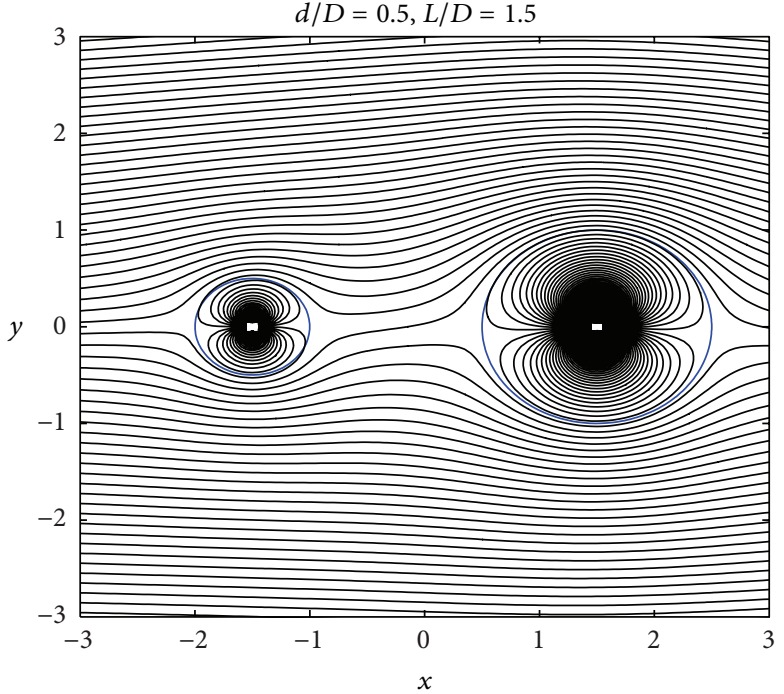

(a)

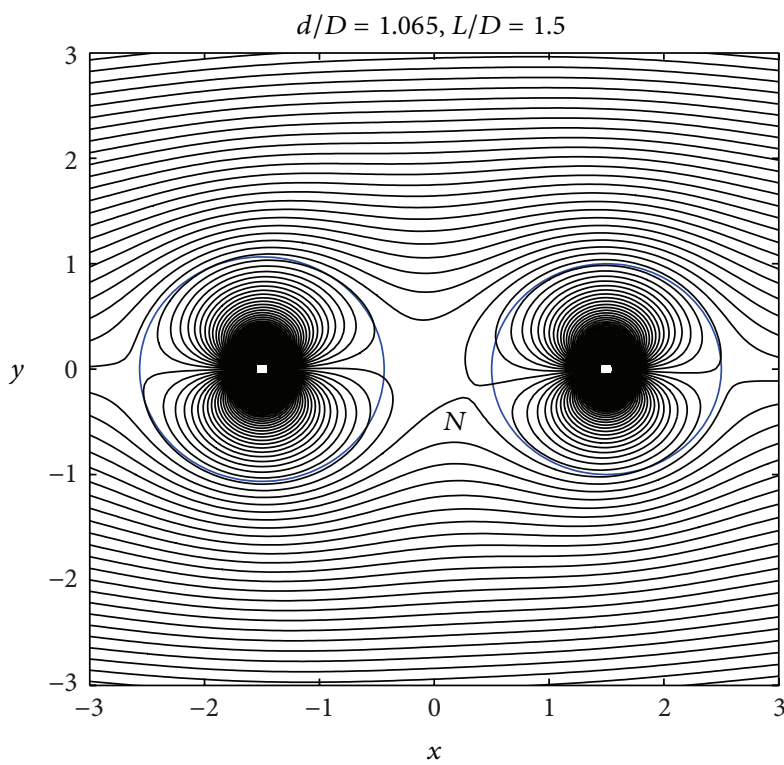

(c)

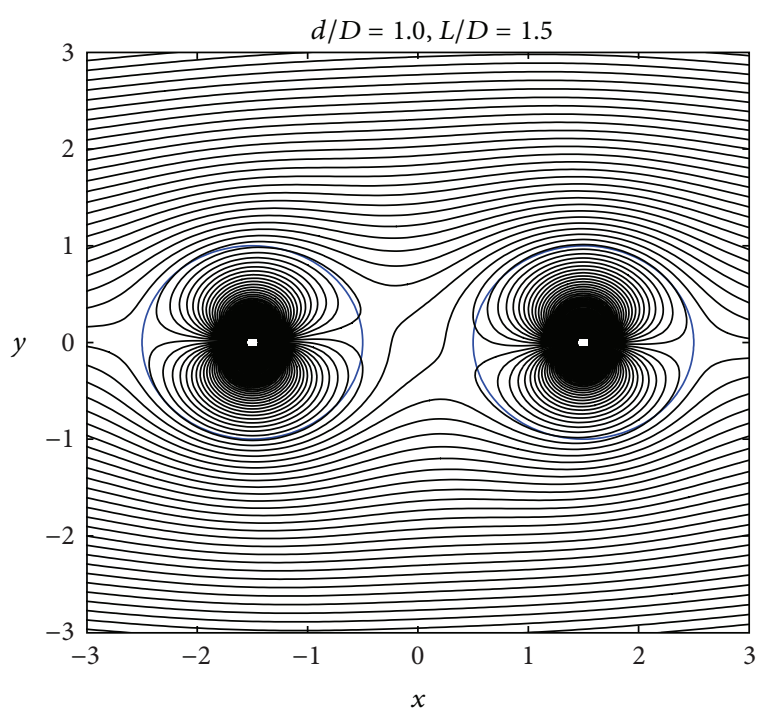

(b)

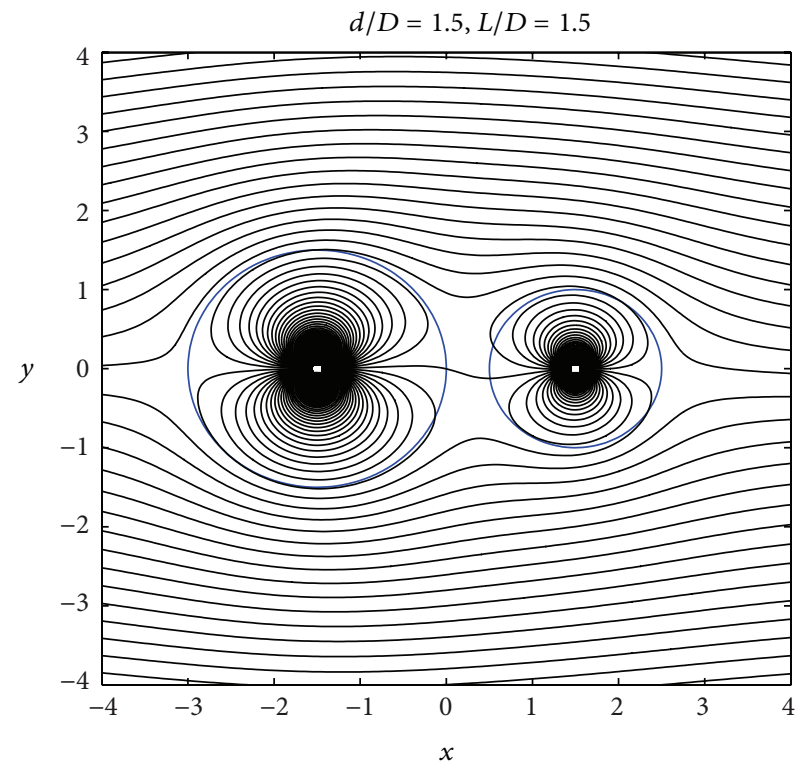

(d)

FIgURE 12: Streamlines around two tandem cylinders with different diameter ratios $d / D$ at $L / D=1.5$.

result of alternating positive and negative circulations of the doublet-pair.

The slope of the diagonally crossing streamline nearest the surface of the upstream cylinder is considered here as a means of evaluating the range of $L / D$ for which the onset of alternating reattachment flow patterns is observed. As shown in Figure 9, the slope $k$ is defined as the slope of the line joining points $P_{1}$ and $P_{2}$, where $P_{1}$ and $P_{2}$ are the horizontal and vertical "intercepts," respectively, of the abovementioned streamline.

The slope $k$ decreases with $L / D$ and depicts an "inflection point" in the vicinity of $L / D=5.5$, as shown in Figure 10. The authors hypothesize that there exists a certain slope $k_{\text {cri }}$ at which there is a transition from the pattern of alternating reattachment to the next pattern where the wakes behind the two cylinders are independent of each other. In other words, when $k \geq k_{\text {cri }}$ (or equivalently $L / D \leq(L / D)_{\text {IIcri }}$ ), the phenomenon of alternating reattachment on the downstream cylinder occurs, and when $k<k_{\text {cri }}$ (or equivalently $L / D>$ $\left.(L / D)_{\text {IIcri }}\right)$, the flow patterns correspond to those of two independent cylinder wakes. It can be seen from Figure 10 that, at $L / D=5.3$, the curve of $k$ versus $L / D$ has a sudden change in slope. One would expect a transition of flow patterns at this critical spacing ratio. This observation is indeed consistent with experimental observations (Igarashi [2]; Zdravkovich [11]).

It can also be deduced from Figure 10 that when $L / D>$ 5.3, the interactions between the two cylinders are rather 
weak and the two cylinder wakes are independent. Short of any other criterion, $L / D=5.3$ may be adopted as the threshold condition for independent wake flow patterns of two equal cylinders in tandem arrangement.

The value of $L / D$ for which the two wake flow patterns are independent of each other would be different for unequal cylinder diameters and different incident flow speed. This situation is deliberated later.

3.2. Cylinder-Pair with Unequal Diameters (Special Case of $V=1 / U=1$ ). In this section, the authors consider the situation where $d / D$ is less than 1 and aim to determine if there is a unique relationship between the diameter ratio and the threshold spacing ratio for the onset of type 2 interference. In order to investigate the relationship between $d / D$ and $L / D$, the authors examined the streamline patterns around a pair of cylinders with various $d / D$ and $L / D$, drawing on the analogy with the potential flow theory as before.

Since the streamlines patterns formed when the positive and negative vortices are reversed are mirror images of each other, only the case with the upstream cylinder having a positive vortex and the downstream cylinder a negative vortex needs to be considered hereafter.

Figure 11 shows the streamlines around two tandem cylinders with different diameter ratios $d / D=1 / 3,2 / 3$, and 1 at a spacing ratio $L / D=1.0$. It can be seen that, at this spacing ratio, the two cylinders behave as a single bluff body in all three cases. Nevertheless, the streamline patterns are different at each diameter ratio, as can be seen from the streamlines in the region between the two cylinders.

When $L / D$ is increased to 1.5 , as shown in Figure 12, different streamline patterns are again observed at different diameter ratios $d / D$. At $d / D=0.5$, the "crossing" pattern between the two cylinders is observed. However, when $d / D$ is increased to 1.065 , the lower streamline shows the tendency of moving upwards but has not yet extended across the axis joining the two cylinder centres. This is similar to the patterns observed between two cylinders with equal diameter at small spacing ratios, whereupon the two cylinders behave as a single bluff body. In summary, it appears that a certain relationship exists between $d / D$ and $L / D$ to account for the same flow patterns between the two tandem cylinders of unequal diameters.

By examining the variations of this threshold spacing ratio $(L / D)_{\text {cri }}$ for different diameter ratios $d / D$ (see Figure 13), it can be seen that the corresponding $(L / D)_{\text {cri }}$ is smaller for unequal cylinder-pairs. That the data by other authors scatter widely at $d / D=1$ is due to the different flow speed which could not be harmonized in this plot.

Figure 13 shows the logarithmic relationship between the threshold spacing ratio $(L / D)_{\text {cri }}$ and diameter ratio $d / D$. Note that $d / D$ is always less than or equal to 1 since $D$ is the larger of the two cylinder diameters. Also included in Figure 13 are the reported values of $(L / D)_{\text {cri }}$ at which type 1 interference is ended, depending on the range of Re. It can be seen that, for the same $U$ (or Re), the threshold spacing ratio $(L / D)_{\text {cri }}$ increases with $d / D$. That is to say, the alternating attachment

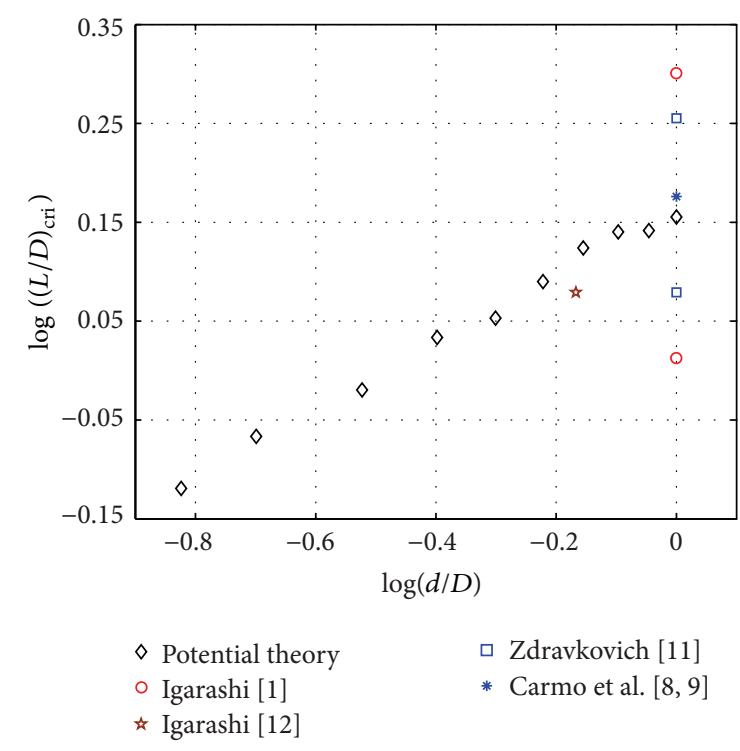

FIGURE 13: The variation of critical spacing ratio $(L / D)_{\text {cri }}$ with diameter ratio $d / D$.

of streamline patterns can be observed at lower centreto-centre spacing ratio $L / D$ for smaller $d / D$, and unequal cylinders should be bundled at closer spacing. Therefore, it can be concluded that in addition to $L / D$, the diameter ratio also has a significant effect on the classification of streamline patterns. The reported data for equal diameter cylinder-pair $(d / D=1)$ are visibly scattered and are logically so because these data are based on different incident flow speed.

The relationship between the critical spacing ratio $(L / D)_{\text {cri }}$ and diameter ratio $d / D$ can be fitted based on Figure 13 as follows:

$$
\log \left(\frac{L}{D}\right)_{\text {cri }}=0.339 \log \left(\frac{d}{D}\right)+0.164
$$

At diameter ratio $d / D=1.0,(L / D)_{\text {cri }}=10^{0.164}=$ 1.458 for the case $V=1 / U=1$, which compares well to the reported results of Carmo et al. [8] or approach the mean value of $(L / D)_{\text {cri }}$ obtained by Igarashi [1] $\left((L / D)_{\mathrm{cri}}=\right.$ 1.03-2, depending on the range of $\mathrm{Re})$ and Zdravkovich [11] $\left((L / D)_{\text {cri }}=1.2-1.8\right.$, depending on the range of Re $)$.

It is noted that (9) may be expressed as follows, approximating the gradient of 0.339 as $1 / 3$ :

$$
\frac{(L / D)}{(d / D)^{1 / 3}}=10^{0.164}=\text { constant }
$$

which indicates the functional relationship, for the special case of $V=1 / U=1.0$; that is,

$$
f\left[\left(\frac{L}{D}\right)\left(\frac{D}{d}\right)^{1 / 3}\right]=\text { constant. }
$$

3.3. Streamline Patterns as a Function of Incident Flow Velocity. In order to investigate the effects of incident flow velocity on the cylinder-pair, the authors examined the streamline 


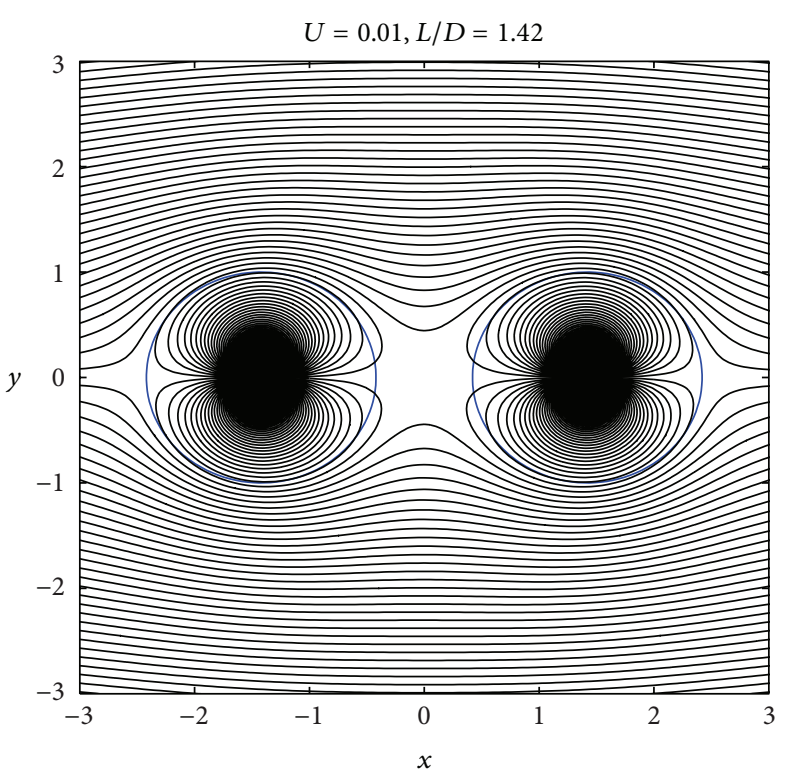

(a)

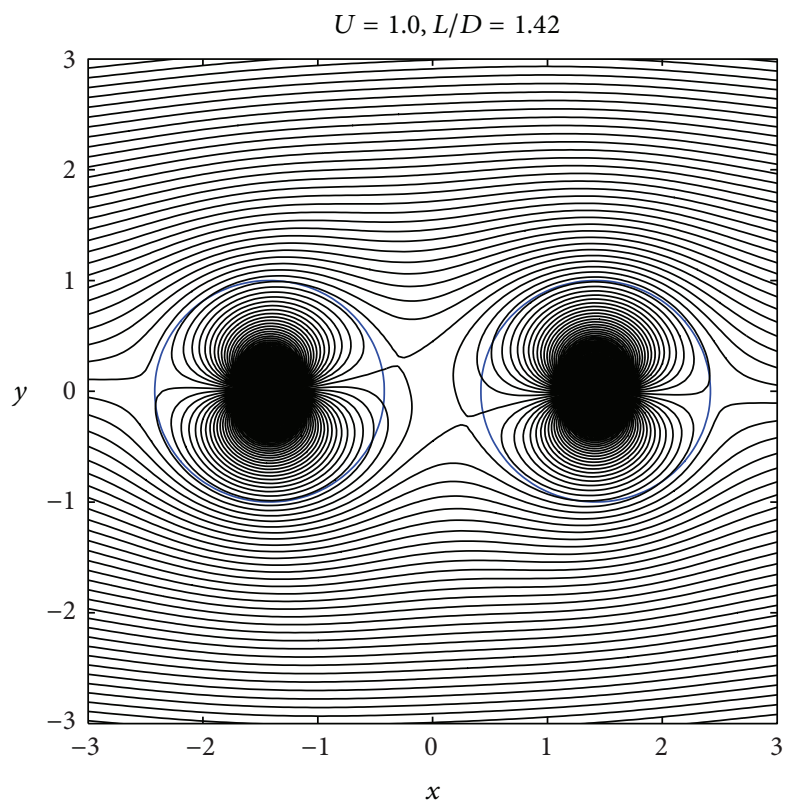

(c)

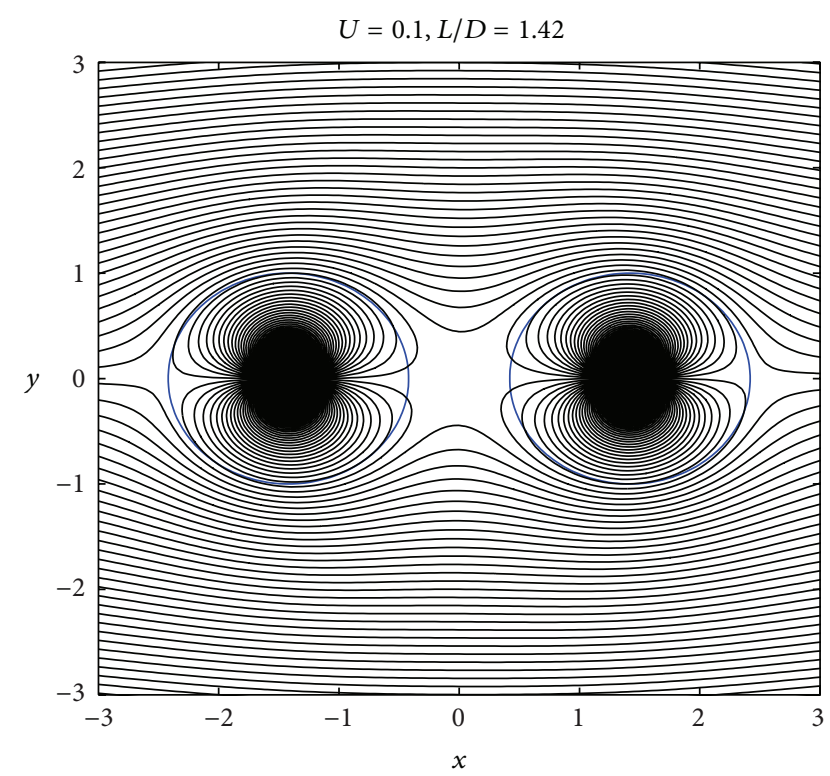

(b)

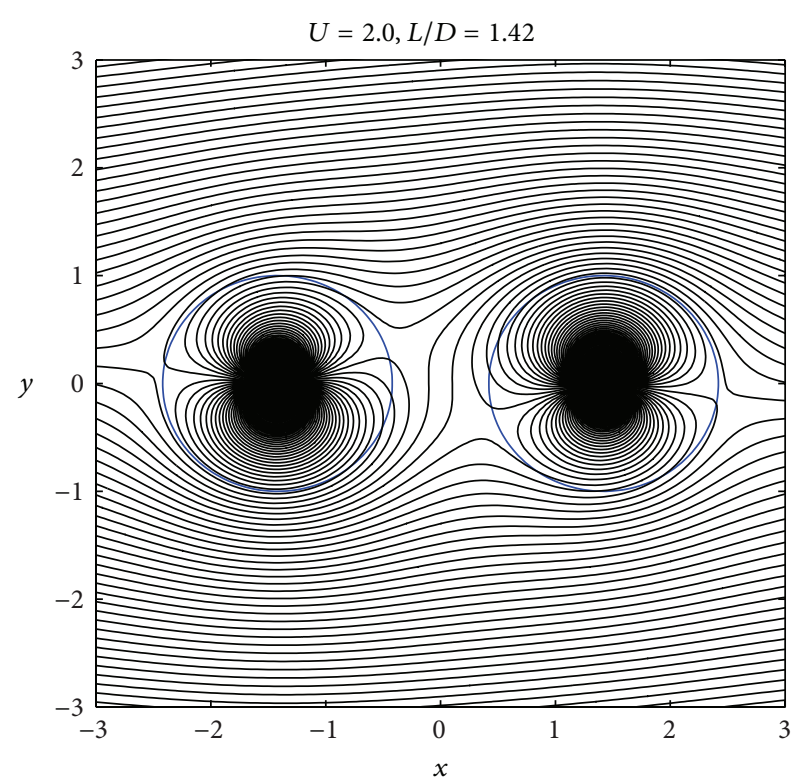

(d)

FIGURE 14: Streamlines around two tandem cylinders with different velocity $V=1 / U$ at $L / D=1.42$.

patterns about the cylinder-pair at various velocities $U(=$ $1 / V$ in the mapping function). As an illustration, the various streamline patterns for $L / D=1.42$ and $d / D=1$ are shown in Figure 14. Different streamline patterns are observed for different $U$ while the spacing and diameter ratios are held constant.

At small $U$ of the order of 0.01-1.0, no "diagonal-crossing" patterns are observed; that is, the two cylinders behave as a single bluff body. However, when $U$ is increased to 2.0, the "crossing" pattern is observed, indicating that the reattachment phenomenon has taken place. The authors repeated the numerical experiment with various diameter ratio $(d / D)$ and flow velocity $U$ and determined the corresponding threshold spacing ratio $(L / D)_{\text {cri }}$. It was observed that $(L / D)_{\text {cri }}$ decreases with $U$ for a fixed $d / D$, but increases with $d / D$ for a fixed velocity. This observation is in good agreement with Igarashi's [1] suggestion that the critical spacing ratio is sensitive to Reynolds number.

Figure 15 shows the variation of the dimensional parameter $(L / D)(D / d)^{1 / 3}$ as a function of dimensionless velocity in the form of a Reynolds number at different diameter ratio $d / D$, where the dimensional parameter $(L / D)(D / d)^{1 / 3}$ is calculated from (10). This Reynolds number is defined as $U(D d)^{1 / 2}$, for which the kinematic viscosity of water is 


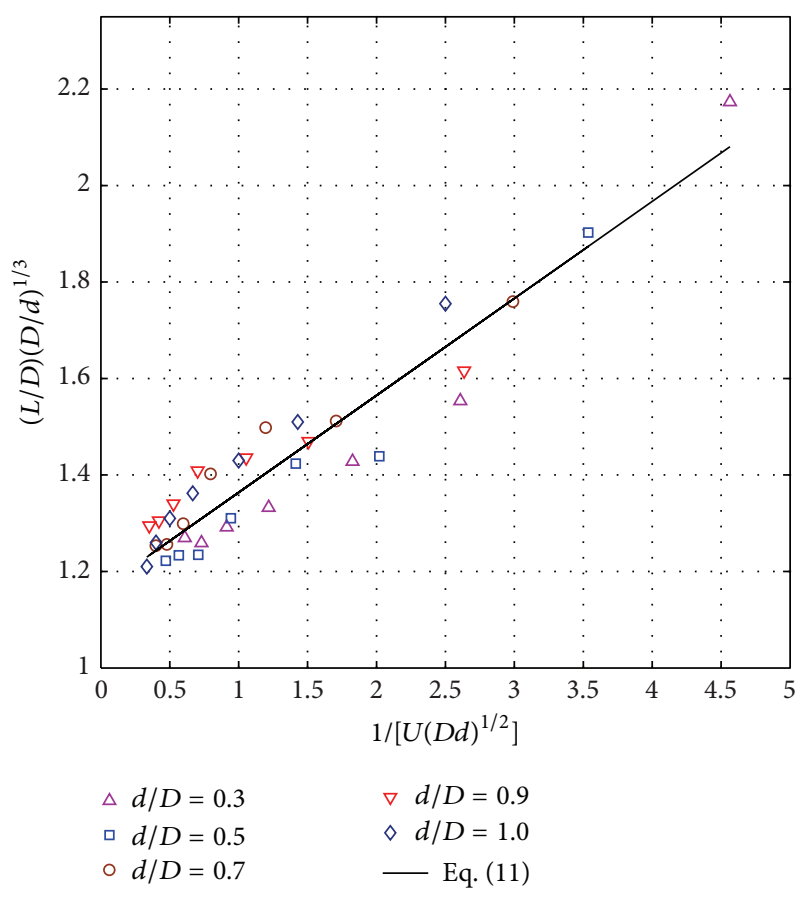

FIGURE 15: Threshold spacing ratio as a function of $U(D d)^{1 / 2}$ at different $d / D$.

constant and left out of the expression without affecting the quality of the presentation. The root mean square value of $D$ and $d$ is chosen as the characteristic length. It can be seen in Figure 15 that the threshold spacing ratio approaches a constant value $(\sim 1.2)$ when $U$ is large. When $U$ is very small, the reattachment phenomenon is not observed even when $L / D$ is large. In terms of physical interpretation, the authors hypothesize that when $U$ is large, the flow is turbulent and reattachment phenomenon would be observed readily. For small $U$, and in the limit when the flow is laminar, the cylinder-pair would behave like a single bluff body.

It can also be seen in Figure 15 that the data collapse and fit into a single curve which is fitted as

$$
\left(\frac{L}{D}\right)\left(\frac{D}{d}\right)^{1 / 3}=\frac{0.201}{U(D d)^{1 / 2}}+1.163
$$

Equation (12) is included in Figure 15 as the solid line.

\section{Conclusions}

Drawing on the analogy of potential flow theory, the flow patterns that are generally seen in experiments for different centre-to-centre spacing ratios and diameter ratios for two cylinders in tandem configuration are reproduced. The authors found that the flow speed $U$, spacing ratio $L / D$, and diameter ratio $d / D$ have significant effects on the flow patterns for flow past a cylinder-pair in tandem arrangement. A cubic relationship $f(L / D, d / D, U)$ of the threshold condition for the wake transition from a single bluff body to a reattachment behavior is established. This study has also found that the occurrence of alternating reattachment phenomenon results from the alternating positive and negative circulations of the doublet-pair. Two cylinders of unequal diameters need to be placed closer to one another (compared to cylinders of equal diameter) in order to avoid the occurrence of vortex shedding between the cylinders.

\section{Conflict of Interests}

The authors declare that there is no conflict of interests regarding the publication of this paper.

\section{Acknowledgments}

This research was supported by the Open Foundation of the State Key Laboratory of Fluid Power Transmission and Control (GZKF-201310), the State Key Laboratory of Satellite Ocean Environment Dynamics (Second Institute of Oceanography, SOA), China, and the National Research Foundation, Singapore (CRP-5-2009-01); funding support from the Maritime and Port Authority of Singapore (MPA) is gratefully acknowledged. Zhiyong Hao also would like to acknowledge the support from Science and Technology Commission of Shanghai Municipality (Pujiang Program, Grant no. 10PJ1404700), Shanghai Education Committee (Program Grant no. 12ZZ149 and J50604), and Shanghai Maritime University (Science \& Technology Program, Grant no. 20100089).

\section{References}

[1] T. Igarashi, "Characteristics of the flow around two circular cylinders arranged in tandem, 1st report," Bulletin of the JSME $B$, vol. 24, no. 188, pp. 323-331, 1981.

[2] T. Igarashi, "Characteristics of the flow around two circular cylinders arranged in tandem, 2nd report," Bulletin of the JSME $B$, vol. 27, no. 233, pp. 2380-2387, 1984.

[3] L. Ljungkrona and B. Sundén, "Flow visualization and surface pressure measurement on two tubes in an inline arrangement," Experimental Thermal and Fluid Science, vol. 6, no. 1, pp. 15-27, 1993.

[4] D. Sumner, S. J. Price, and M. P. Païdoussis, “Tandem cylinders in impulsively started flow," Journal of Fluids and Structures, vol. 13, no. 7-8, pp. 955-965, 1999.

[5] J.-C. Lin, Y. Yang, and D. Rockwell, "Flow past two cylinders in tandem: instantaneous and averaged flow structure," Journal of Fluids and Structures, vol. 16, no. 8, pp. 1059-1071, 2002.

[6] G. Xu and Y. Zhou, "Strouhal numbers in the wake of two inline cylinders," Experiments in Fluids, vol. 37, no. 2, pp. 248-256, 2004.

[7] Y. Zhou and M. W. Yiu, "Flow structure, momentum and heat transport in a two-tandem-cylinder wake," Journal of Fluid Mechanics, vol. 548, pp. 17-48, 2006.

[8] B. S. Carmo, J. R. Meneghini, and S. J. Sherwin, "Possible states in the flow around two circular cylinders in tandem with separations in the vicinity of the drag inversion spacing," Physics of Fluids, vol. 22, no. 5, Article ID 054101, 2010.

[9] B. S. Carmo, J. R. Meneghini, and S. J. Sherwin, "Secondary instabilities in the flow around two circular cylinders in tandem," Journal of Fluid Mechanics, vol. 644, pp. 395-431, 2010. 
[10] D. Sumner, "Two circular cylinders in cross-flow: a review," Journal of Fluids and Structures, vol. 26, no. 6, pp. 849-899, 2010.

[11] M. M. Zdravkovich, "The effects of interference between circular cylinders in cross flow," Journal of Fluids and Structures, vol. 1, no. 2, pp. 239-261, 1987.

[12] T. Igarashi, "Characteristics of the flow around two circular cylinders of different diameters arranged in tandem," Bulletin of the JSME B, vol. 25, no. 201, pp. 349-357, 1982.

[13] M. Mahbub Alam and Y. Zhou, "Strouhal numbers, forces and flow structures around two tandem cylinders of different diameters," Journal of Fluids and Structures, vol. 24, no. 4, pp. 505-526, 2008.

[14] M. Zhao, L. Cheng, B. Teng, and D. F. Liang, "Numerical simulation of viscous flow past two circular cylinders of different diameters," Applied Ocean Research, vol. 27, no. 1, pp. 39-55, 2005.

[15] M. Zhao, L. Cheng, B. Teng, and G. Dong, "Hydrodynamic forces on dual cylinders of different diameters in steady currents," Journal of Fluids and Structures, vol. 23, no. 1, pp. 59-83, 2007.

[16] Y. Gao, S. Etienne, D. Yu, X. Wang, and S. Tan, "Bi-stable flow around tandem cylinders of different diameters at low Reynolds number," Fluid Dynamics Research, vol. 43, no. 5, Article ID 055506, 2011.

[17] Y. Gao, S. Tan, E. Stephane, and X. Wang, "Experimental study on the flow around two tandem cylinders of different diameters," Internal Report of MRC, 2012.

[18] A. Millward, D. Nicolaou, and S. G. Rigby, "Numerical modeling of the water flow around a fast ship with a transom stern," International Journal of Maritime Engineering, The Royal Institute of Naval Architects, pp. 181-194, 2003.

[19] M. Shahjada Tarafder and K. Suzuki, "Numerical calculation of free-surface potential flow around a ship using the modified Rankine source panel method," Ocean Engineering, vol. 35, no. 5-6, pp. 536-544, 2008.

[20] Y. Ahmed and C. Guedes Soares, "Simulation of free surface flow around a VLCC hull using viscous and potential flow methods," Ocean Engineering, vol. 36, no. 9-10, pp. 691-696, 2009.

[21] E. R. Johnson and N. R. McDonald, "The motion of a vortex near two circular cylinders," Proceedings of The Royal Society of London A, vol. 460, no. 2044, pp. 939-954, 2004.

[22] D. A. Burton, J. Gratus, and R. W. Tucker, "Hydrodynamic forces on two moving discs," Theoretical and Applied Mechanics, vol. 31, no. 2, pp. 153-188, 2004.

[23] D. G. Crowdy, "Analytical solutions for uniform potential flow past multiple cylinders," European Journal of Mechanics B, vol. 25, no. 4, pp. 459-470, 2006.

[24] D. J. Acheson, Elementary Fluid Dynamics, Oxford University Press, Oxford, UK, 1990. 


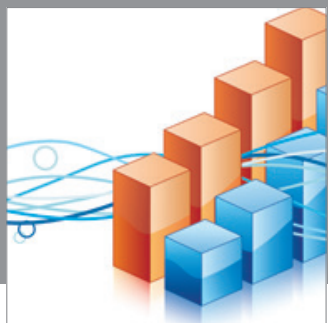

Advances in

Operations Research

mansans

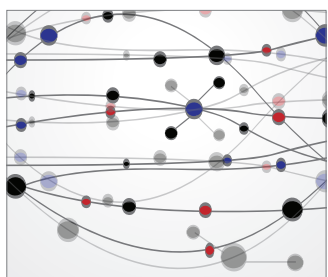

The Scientific World Journal
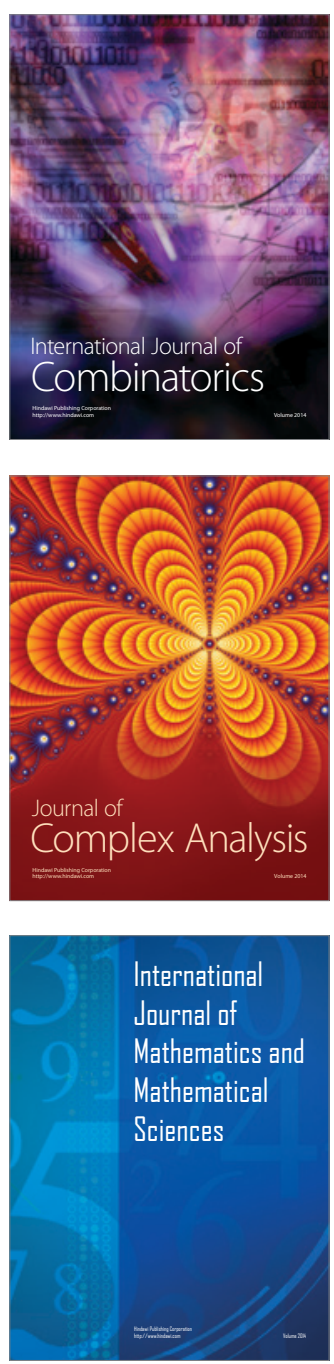
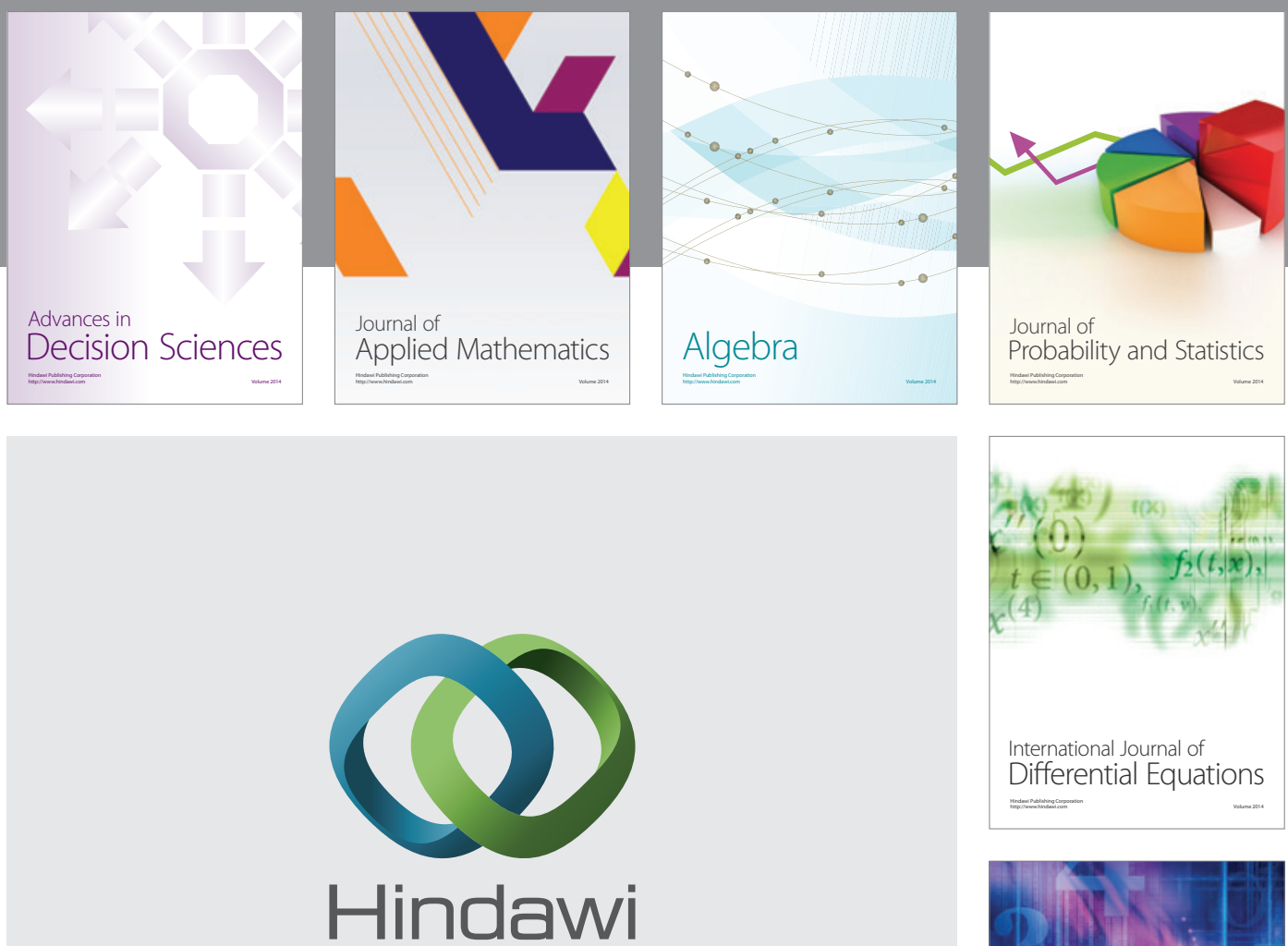

Submit your manuscripts at http://www.hindawi.com
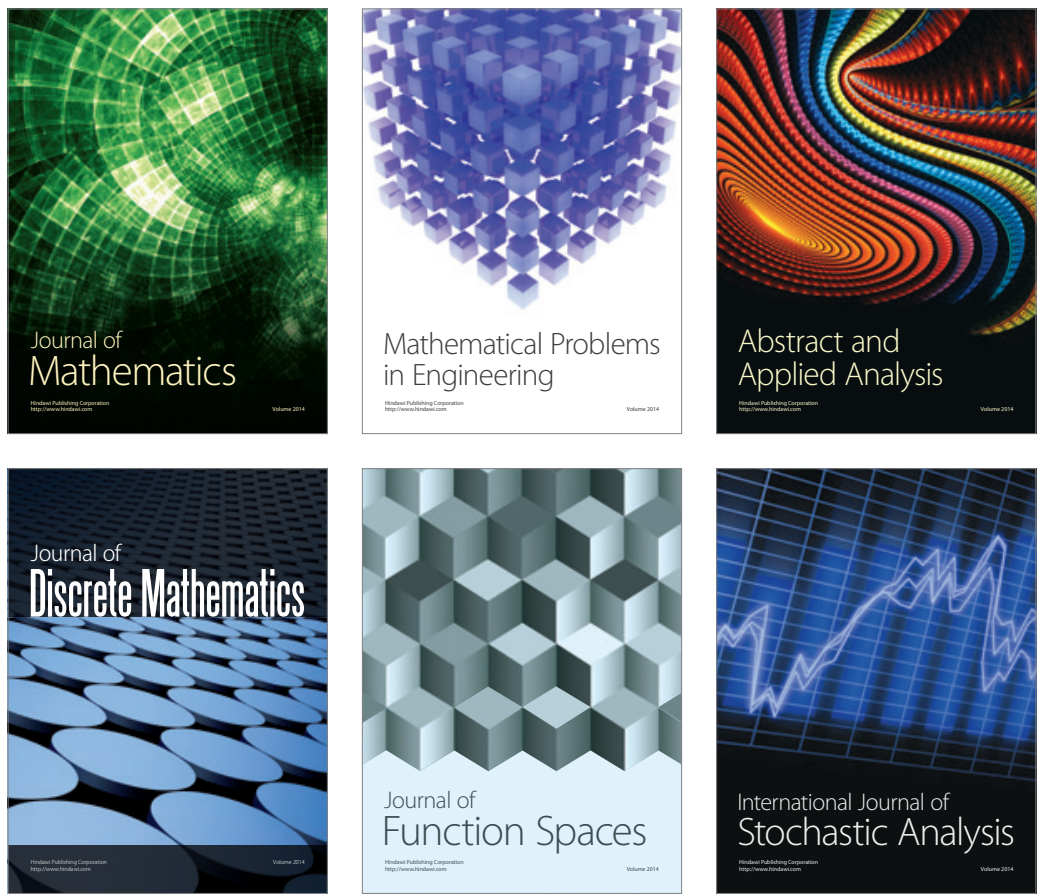

Journal of

Function Spaces

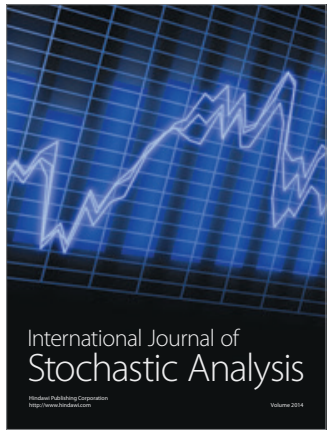

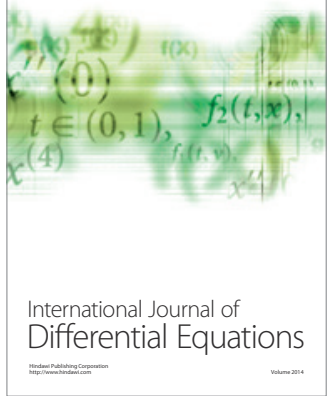
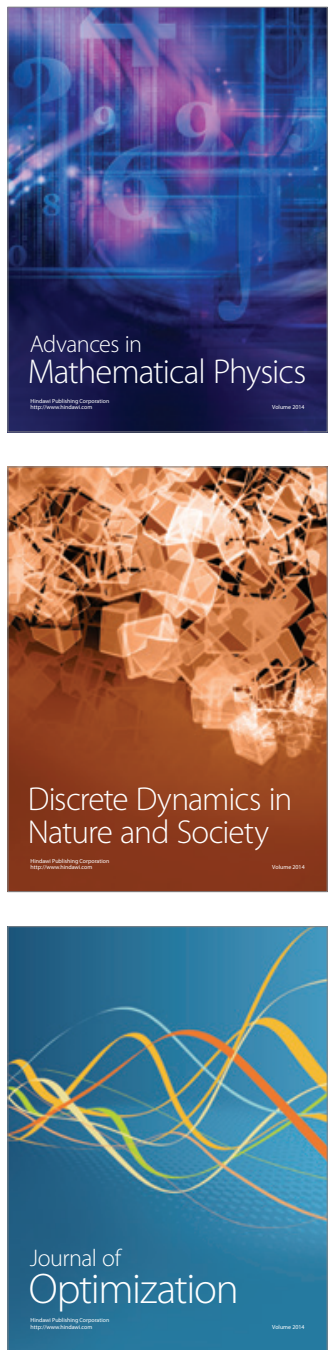